(c) American Dairy Science Association, 2005.

\title{
Acid and Bile Tolerance and Cholesterol Removal Ability of Lactobacilli Strains
}

M. T. Liong and N. P. Shah

School of Molecular Sciences, Victoria University, Werribee Campus, PO Box 14428, Victoria 8001, Australia

\begin{abstract}
Eleven strains of lactobacilli were studied for their acid and bile tolerance. Possible mechanisms of cholesterol removal by strains of lactobacilli were examined. Cholesterol assimilation as determined by the difference in cholesterol content in the medium before and after the incubation period showed that all lactobacilli strains were able to assimilate cholesterol at varying levels ranging from 12.03 to $32.25 \mu \mathrm{g} / \mathrm{mL}$. Cholesterol removal was associated with growth of cultures. Binding of cholesterol to lactobacilli cells was determined using growing, heat-killed, and resting cells in phosphate buffer. Cholesterol removed by dead and resting cells ranged from 0.79 to $3.82 \mathrm{mg} / \mathrm{g}$ of dry weight compared with growing cells, which ranged from 4.53 to $16.03 \mathrm{mg} / \mathrm{g}$ of dry weight. Fatty acid methyl esters, as quantified using gas chromatography, showed changes in lipid profiles in cells grown in the presence of cholesterol compared with those grown without cholesterol. Fatty acid profiles, especially of hexadecanoic, octadecanoic, total saturated, and unsaturated acids suggested that cholesterol from the medium was incorporated into the cellular membrane. These findings suggest that strains of lactobacilli could remove cholesterol via various mechanisms and may be promising candidates for use as a dietary adjunct to lower serum cholesterol in vivo.
\end{abstract}

(Key words: lactobacilli, acid tolerance, bile tolerance, cholesterol assimilation)

Abbreviation key: LDL = low density lipoprotein, MRS = de Man, Rogosa, Sharpe medium.

\section{INTRODUCTION}

Studies have shown that elevated serum cholesterol is associated with increased risk of coronary heart disease. For each $1 \mathrm{mmol}$ above the normal cholesterol

Received January 15, 2004.

Accepted August 10, 2004

Corresponding author: Nagendra P. Shah; e-mail: nagendra.shah @vu.edu.au. level, the risk of coronary heart disease was approximately 35\% higher, and coronary death was $45 \%$ higher. Even a small reduction in serum cholesterol of $1 \%$ was found to reduce the risk of coronary heart disease by 2 to 3\% (Manson et al., 1992). Consumption of dairy products containing probiotics has been proposed as a means to lower serum cholesterol (Greenwald, 1991).

Probiotics are defined as a "live microbial supplement that beneficially affects the host animal by improving its intestinal microbial balance" (Fuller, 1992). To provide health benefits, probiotics must overcome physical and chemical barriers such as acid and bile in the gastrointestinal tract (Gibson et al., 2000). Milk fermented with lactobacilli was first demonstrated to exhibit hypocholesterolemic effects in humans as early as 1963 (Shaper et al., 1963; Mann, 1974). Various studies have shown that some lactobacilli could lower total cholesterol and low-density lipoprotein (LDL) cholesterol (Anderson and Gilliland, 1999; Sanders, 2000).

The exact mechanisms of serum cholesterol reduction by probiotic bacteria are unclear. Some strains of Lactobacillus acidophilus were found to secrete bile salt hydrolase (cholylglycine hydrolase; EC 3.5.1.24), which catalyzes the hydrolysis of glycine- or taurine-conjugated bile salts into amino acid residues and free bile salts (bile acids; Corzo and Gilliland, 1999). Free bile salts are less soluble than conjugated bile salts, resulting in lower absorption in the intestinal lumen. Deconjugation of bile acids can reduce serum cholesterol levels by increasing the formation of new bile acids that are needed to replace those that have escaped the enterohepatic circulation (Reynier et al., 1981). Another mechanism for cholesterol reduction was proposed by Noh et al. (1997), who suggested that $L$. acidophilus incorporates some of the cholesterol removed from the medium into the cellular membranes during growth. Cholesterol incorporated into or adhered to the bacterial cells would be less available for absorption from the intestine into the blood. Cholesterol incorporated into the cells of $L$. acidophilus was postulated to alter the cellular membrane or cell wall of the organism. Gram staining of $L$. acidophilus showed that only a proportion of cells grown in the presence of cholesterol 
stained gram-positive, whereas all those grown without cholesterol stained gram-positive. However, this was debated as experiments using membrane isolated from strains of Lactobacillus casei grown in broth containing cholesterol revealed no measurable incorporation of cholesterol (Brashears et al., 1998).

Dambekodi and Gilliland (1998) have shown no relationship between the amount of cholesterol removal in vitro and the degree of bile salt deconjugation, which led to another hypothesis that cholesterol removal may be related to assimilation of cholesterol. In vitro experiments showed that strains of lactobacilli that were able to assimilate cholesterol were also able to reduce cholesterol in vivo. Cholesterol assimilation was associated with the presence of bile salts, and cholesterol removal from the medium increased with increasing concentration of bile salt (Rasic et al., 1992; Tahri et al., 1996). However, bile tolerant strains of $L$. acidophilus did not exhibit the greatest ability to assimilate cholesterol; strains that possessed minimal bile tolerance actually assimilated more cholesterol (Dambekodi and Gilliland, 1998). Cholesterol assimilation by strains of $L$. acidophilus during refrigerated storage of nonfermented milk suggested that cholesterol uptake was associated with bacterial growth and viability (Piston and Gilliland, 1994). Similarly, Pereira and Gibson (2002) suggested that the cholesterol assimilation ability of the bacteria was growth dependent.

The aims of this study were to investigate the probiotic properties (acid and bile tolerance) of 11 lactobacilli strains and their cholesterol removal abilities to understand the possible mechanisms of cholesterol removal.

\section{MATERIALS AND METHODS}

\section{Bacteria}

Seven strains of Lactobacillus casei were obtained from the Victoria University Culture Collection (Werribee, Australia). Lactobacillus casei CSCC 2607 was originally obtained from the Commonwealth Scientific and Industrial Organization (CSIRO) (Highett, Australia); L. casei ASCC 1520, L. casei ASCC 1521, L. casei ASCC 279, L. casei ASCC 290, L. casei ASCC 292 and L. casei ATCC 15820 were obtained from the Australia Starter Culture Collection Center (ASCC, Werribee, Australia). Four strains of Lactobacillus acidophilus were obtained from ASCC. All strains were of human origin. Stock cultures were stored in $40 \%$ glycerol at $-80^{\circ} \mathrm{C}$. The organisms were subcultured 3 times before use in sterile de Man, Rogosa, Sharpe (MRS) broth using $1 \%$ inoculum and incubation for $20 \mathrm{~h}$ at $37^{\circ} \mathrm{C}$.

\section{Acid Tolerance}

Acid tolerance of the cultures was studied by incubating the organisms in MRS broth supplemented with $0.30 \%$ oxgall. The $\mathrm{pH}$ was adjusted to 2.0 with $\mathrm{HCl}$ and cultures were incubated at $37^{\circ} \mathrm{C}$ for $2 \mathrm{~h}$. Each of the 11 strains of $L$. acidophilus and $L$. casei was subcultured at least 3 times before experimental use, followed by centrifugation after the final subculture, inoculation (10\% vol/vol) into the broth, and growth monitoring using the plate count method. Bacilli generally divide in one plane, but can produce chains of cells due to incomplete separation of divided cells. Thus, fermentation broth containing probiotic cultures was sonicated for $5 \mathrm{~s}$ to disrupt clumps of lactobacilli (Bermudez et al., 2001) before serial dilutions were performed. Subsequent serial dilution blanks were vortexed for $30 \mathrm{~s}$ individually. A 1-mL sample was taken every $30 \mathrm{~min}$ for 2 $\mathrm{h}$, and 10 -fold serial dilutions were made using peptone water diluent. Samples were plated onto MRS agar, and the plates were incubated at $37^{\circ} \mathrm{C}$ for $24 \mathrm{~h}$ in an anaerobic jar (Becton Dickinson Microbiology Systems, Sparks, MD) with a Gas Generating Kit (Oxoid, Ltd., West Heidelberg, Victoria, Australia). Acid tolerance was determined by comparing the final plate count after $2 \mathrm{~h}$ with the initial plate count at $0 \mathrm{~h}$. The experiments were repeated twice.

\section{Bile Tolerance}

Three types of bile were used, namely oxgall, cholic acid, and taurocholic acid, to study bile tolerance of the organisms. Bile tolerance was studied according to the method of Gilliland and Walker (1990). Briefly, MRS broth containing $0.30 \%$ (wt/vol) of oxgall, cholic acid, or taurocholic acid was inoculated with each strain, and incubated at $37^{\circ} \mathrm{C}$. The control comprised MRS broth without bile salt. According to the material safety data sheet and Merck solubility index, cholic acid has a solubility of $0.28 \mathrm{~g} / \mathrm{L}$ in water at $15^{\circ} \mathrm{C}$, whereas taurocholic acid is completely soluble in water. Bacterial growth was monitored by measuring absorbance with a spectrophotometer (Pharmacia Novaspec II, Cambridge, UK) at $620 \mathrm{~nm}$ at hourly intervals for 7 to $8 \mathrm{~h}$. The absorbance values obtained were plotted against the incubation time, and the bile tolerance of each strain was based on the time required for the absorbance value to increase by 0.3 unit. $\mathrm{pH}$ values of all the fermentation broths at time $=0$ were measured, and another measurement was taken after the absorbance increased by 0.3 unit. All experiments were replicated twice.

\section{Cholesterol Removal}

Freshly prepared MRS broth was supplemented with $0.30 \%$ oxgall as a bile salt. Water-soluble cholesterol 
Table 1. Effect of $\mathrm{pH} 2.0$ on viability of Lactobacillus acidophilus and L. casei.

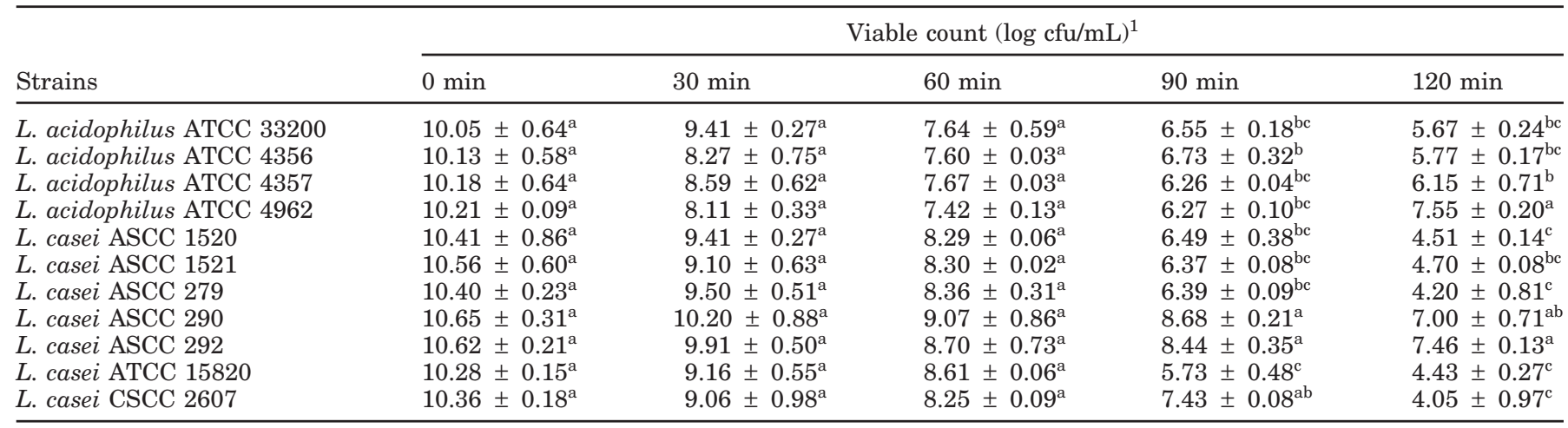

${ }^{\mathrm{abc}}$ Means in the same column followed by different superscript letters are significantly different $(P<0.05)$.

${ }^{1}$ Results are expressed as mean $\pm \mathrm{SEM}$; each data point is the average of 2 repeated measurements from 2 independently replicated experiments, $\mathrm{n}=2$.

(polyoxyethanyl cholesteryl sebacate) was filter-sterilized and added to the broth at a final concentration of 70 to $100 \mu \mathrm{g} / \mathrm{mL}$, inoculated with each strain (at $1 \%$ ), and incubated anaerobically at $37^{\circ} \mathrm{C}$ for $20 \mathrm{~h}$. After the incubation period, cells were centrifuged and the remaining cholesterol concentration in the broth was determined using a modified colorimetric method as described by Rudel and Morris (1973). One milliliter of the aliquot was added with $1 \mathrm{~mL}$ of $\mathrm{KOH}(33 \% \mathrm{wt} / \mathrm{vol})$ and $2 \mathrm{~mL}$ of absolute ethanol, vortexed for $1 \mathrm{~min}$, and heated at $37^{\circ} \mathrm{C}$ for $15 \mathrm{~min}$. After cooling, $2 \mathrm{~mL}$ of distilled water and $3 \mathrm{~mL}$ of hexane were added and vortexed for $1 \mathrm{~min}$. One milliliter of the hexane layer was transferred into a glass tube and evaporated under nitrogen. The residue was immediately dissolved in $2 \mathrm{~mL}$ of o-phthalaldehyde reagent. After complete mixing, 0.5 $\mathrm{mL}$ of concentrated sulfuric acid was added and the mixture was vortexed for $1 \mathrm{~min}$. Absorbance was read at $550 \mathrm{~nm}$ (Pharmacia Novaspec II) after $10 \mathrm{~min}$. All experiments were replicated twice.

\section{Cholesterol Removal by Dead and Resting Cells}

Freshly prepared MRS broth containing $0.30 \%$ oxgall was inoculated with each strain of lactobacilli and incubated at $37^{\circ} \mathrm{C}$ for $20 \mathrm{~h}$. Cells were harvested after the incubation period by centrifuging at 10,000 $\times g$ (Microspin 24, Sorvall Instruments, Melbourne, Australia) at $4^{\circ} \mathrm{C}$ for $10 \mathrm{~min}$. The cell pellet was washed twice with sterile distilled water. For preparation of heat-killed cells, the cell pellet was suspended in $10 \mathrm{~mL}$ of sterile distilled water and autoclaved for $15 \mathrm{~min}$ at $121^{\circ} \mathrm{C}$. The heat-killed cells were suspended in MRS broth containing $0.30 \%$ oxgall and water-soluble cholesterol. For preparation of resting cells, the cell pellet was suspended in $10 \mathrm{~mL}$ of sterile $0.05 M$ phosphate buffer ( $\mathrm{pH}$ 6.8) containing oxgall and water-soluble cholesterol
(Kimoto et al., 2002). All strains were incubated at $37^{\circ} \mathrm{C}$ for $20 \mathrm{~h}$. The spent broth was assayed for cholesterol content as mentioned above. Cholesterol assimilation by growing, resting, and dead cells was expressed in dry weight to obtain uniformity in all treatments. The following equation was used:

$$
\text { Cholesterol assimilation }=\left(\mathrm{C}_{1}-\mathrm{C}_{2}\right) /\left(\mathrm{W}_{2}-\mathrm{W}_{1}\right),
$$

where $\mathrm{C}_{1}$ and $\mathrm{C}_{2}$ were the amount of cholesterol present in the fermentation broths at time $=0$ and $20 \mathrm{~h}$, respectively, and $\mathrm{W}_{1}$ and $\mathrm{W}_{2}$ were the dry weight of the individual culture at time $=0$ and $20 \mathrm{~h}$, respectively, for all treatments studied. The experiments were repeated twice.

\section{Cellular Fatty Acids Composition}

Cellular lipids were extracted using a modified method of Murga et al. (2000). The cell pellet was mixed with $1 \mathrm{~mL}$ of methanol: chloroform $(2: 1, \mathrm{vol} / \mathrm{vol})$, and vortexed at room temperature for $2 \mathrm{~min}$. Aliquots were left to stand for $24 \mathrm{~h}$ at $4^{\circ} \mathrm{C}$ and centrifuged $(10,000 \times$ g, $10 \mathrm{~min}, 4^{\circ} \mathrm{C}$ ). The supernatant was collected and the cell pellet was washed twice with $1 \mathrm{~mL}$ of methanol: chloroform: distilled water (2:1:0.8). All 3 supernatants were pooled and vortexed for $1 \mathrm{~min}$. To this, $1 \mathrm{~mL}$ each of distilled water and chloroform were added, followed by vortexing for $2 \mathrm{~min}$. The mixture was allowed to separate and the chloroform layer (bottom) was collected and evaporated. The lipid residue was dissolved in $0.5 \mathrm{~mL}$ of hexane and converted into fatty acid methyl esters with the addition of $100 \mu \mathrm{L}$ of methanolic $\mathrm{KOH}$ $(2 M)$. Hydrochloric acid ( $2 M$ ) was added until methyl orange indicator changed to pink and the mixture was allowed to settle. The organic layer $(10 \mu \mathrm{L})$ was collected and injected into a gas chromatograph (Varian Star 
3400 CX, Walnut Creek, CA), equipped with a flame ionization detector. A stainless steel column $(30 \mathrm{~m} \times$ $0.25 \mathrm{~mm}$ ) packed with $70 \%$ cyanopropyl polysilphenylene siloxane was used. The oven temperature was held at $100^{\circ} \mathrm{C}$ after sample injection and increased to $225^{\circ} \mathrm{C}$ with a rate of $5^{\circ} \mathrm{C} / \mathrm{min}$. The injector and detector temperatures were $260^{\circ} \mathrm{C}$ and $280^{\circ} \mathrm{C}$, respectively. Helium was used as the carrier gas ( $3 \mathrm{~mL} / \mathrm{min})$, split ratio 1:100 and the injection volume was $1 \mu \mathrm{L}$. The concentration of fatty acid methyl esters in samples was determined using standards (Sigma Chemical Co., St. Louis, MO). All experiments were repeated twice.

\section{Statistical Analysis}

Data analysis was carried out with SPSS Inc. software (version 10.0; SPSS Inc., Chicago, IL). One-way ANOVA was used to study significant difference between means, with significance level at $\alpha=0.05$. Tukey's test was used to perform multiple comparisons between means. All data are presented as mean \pm standard error of means. The acid tolerance experiment and cellular fatty acid profiles were independently replicated 2 times $(\mathrm{n}=2)$, with 2 measurements per replicate. The mean of the repeated measurements yielded the value for each replicate. The bile tolerance and cholesterol assimilation experiments were independently replicated 2 times $(n=2)$, with 3 measurements per replicate. The mean of the repeated measurements yielded the value for each replicate.

\section{RESULTS}

\section{Acid Tolerance of Lactobacilli}

The effect of acid on the viability of lactobacilli is shown in Table 1. All strains showed tolerance to $\mathrm{pH}$ 2.0 for $2 \mathrm{~h}$ despite variations in the degree of viability. L. acidophilus ATCC 4962, L. casei ASCC 290 and $L$. casei ASCC 292 were the most acid-tolerant strains, with more than $10^{7} \mathrm{cfu} / \mathrm{mL}$ after incubation for $2 \mathrm{~h}$ at pH 2.0, while L. casei ASCC 1520, L. casei ASCC 1521, L. casei ASCC 279, L. casei ATCC 15820 and L. casei CSCC 2607 were the most acid-sensitive strains, with only $10^{4}$ total $\mathrm{cfu} / \mathrm{mL}$ after the 2 -h incubation. In general, there was a greater reduction in total colony-forming units for the strains of $L$. acidophilus compared with those of $L$. casei for the first hour of incubation; the former decreased by 2.41 to $2.79 \mathrm{log}$ cycles, whereas the latter decreased by 1.58 to $2.26 \mathrm{log}$ cycles. However, strains of $L$. acidophilus showed greater acid tolerance over the entire incubation period, and their counts decreased by 2.66 to 4.38 log cycles, compared with 3.16 to 6.20 log cycles for $L$. casei. For acid-sensitive strains, the viability decreased slowly for the first hour of incu- 
Table 3. Cholesterol assimilation (in $\mu \mathrm{g} / \mathrm{mL}$ ) by lactobacilli in different bile media. ${ }^{1}$

\begin{tabular}{|c|c|c|c|c|}
\hline Strains & MRS broth & $\begin{array}{l}\text { MRS broth }+ \\
0.3 \% \text { oxgall }\end{array}$ & $\begin{array}{l}\text { MRS broth }+ \\
0.3 \% \text { cholic acid }\end{array}$ & $\begin{array}{l}\text { MRS broth }+0.3 \% \\
\text { taurocholic acid }\end{array}$ \\
\hline L. acidophilus ATCC 33200 & $21.61 \pm 0.19^{\mathrm{a}, \mathrm{BC}}$ & $22.09 \pm 0.21^{\mathrm{cd}, \mathrm{AB}}$ & $23.80 \pm 0.52^{\mathrm{c}, \mathrm{A}}$ & $20.80 \pm 0.25^{\mathrm{a}, \mathrm{C}}$ \\
\hline L. acidophilus ATCC 4356 & $20.52 \pm 0.46^{\mathrm{ab}, \mathrm{A}}$ & $21.78 \pm 0.23^{\mathrm{d}, \mathrm{A}}$ & $14.01 \pm 0.14^{\mathrm{d}, \mathrm{C}}$ & $9.07 \pm 0.30^{\mathrm{e}, \mathrm{D}}$ \\
\hline L. acidophilus ATCC 4357 & $21.30 \pm 0.26^{\mathrm{a}, \mathrm{A}}$ & $19.65 \pm 0.23^{\mathrm{e}, \mathrm{B}}$ & $14.32 \pm 0.13^{\mathrm{d}, \mathrm{D}}$ & $16.88 \pm 0.31^{\mathrm{b}, \mathrm{C}}$ \\
\hline L. acidophilus ATCC 4962 & $19.22 \pm 0.47^{\mathrm{bc}, \mathrm{C}}$ & $23.40 \pm 0.39^{\mathrm{cd}, \mathrm{B}}$ & $27.60 \pm 0.25^{\mathrm{b}, \mathrm{A}}$ & $14.69 \pm 0.25^{\mathrm{c}, \mathrm{D}}$ \\
\hline L. casei ASCC 1520 & $20.68 \pm 0.21^{\mathrm{ab}, \mathrm{C}}$ & $32.25 \pm 0.42^{\mathrm{a}, \mathrm{B}}$ & $34.69 \pm 0.45^{\mathrm{a}, \mathrm{A}}$ & $8.86 \pm 0.27^{\mathrm{ef}, \mathrm{D}}$ \\
\hline L. casei ASCC 1521 & $18.02 \pm 0.44^{\mathrm{cd}, \mathrm{C}}$ & $18.71 \pm 0.40^{\mathrm{e}, \mathrm{BC}}$ & $29.64 \pm 0.35^{\mathrm{b}, \mathrm{A}}$ & $20.32 \pm 0.31^{\mathrm{a}, \mathrm{B}}$ \\
\hline L. casei ASCC 279 & $13.07 \pm 0.25^{\mathrm{e}, \mathrm{B}}$ & $12.03 \pm 0.58^{\mathrm{f}, \mathrm{B}}$ & $20.57 \pm 0.55^{\mathrm{d}, \mathrm{A}}$ & $3.76 \pm 0.24^{\mathrm{g}, \mathrm{C}}$ \\
\hline L. casei ASCC 290 & $18.75 \pm 0.46^{\mathrm{bc}, \mathrm{B}}$ & $12.66 \pm 0.42^{\mathrm{f}, \mathrm{C}}$ & $23.02 \pm 0.64^{\mathrm{c}, \mathrm{A}}$ & $6.93 \pm 0.26^{\mathrm{f}, \mathrm{D}}$ \\
\hline L. casei ASCC 292 & $16.67 \pm 0.17^{\mathrm{d}, \mathrm{B}}$ & $29.33 \pm 0.16^{\mathrm{b}, \mathrm{A}}$ & $34.53 \pm 0.35^{\mathrm{a}, \mathrm{A}}$ & $12.14 \pm 0.17^{\mathrm{d}, \mathrm{B}}$ \\
\hline L. casei ATCC 15820 & $16.04 \pm 0.44^{\mathrm{d}, \mathrm{C}}$ & $23.97 \pm 0.37^{\mathrm{c}, \mathrm{B}}$ & $28.80 \pm 0.22^{\mathrm{b}, \mathrm{A}}$ & $11.93 \pm 0.85^{\mathrm{d}, \mathrm{D}}$ \\
\hline L. casei CSCC 2607 & $10.00 \pm 0.44^{\mathrm{f}, \mathrm{B}}$ & $20.38 \pm 0.31^{\mathrm{de}, \mathrm{A}}$ & $10.94 \pm 0.32^{\mathrm{e}, \mathrm{B}}$ & $7.82 \pm 0.35^{\mathrm{ef}, \mathrm{C}}$ \\
\hline
\end{tabular}

${ }_{\text {abcdef Means in the same column followed by different lowercase letters are significantly different }(P<}$ $0.05)$.

${ }^{\mathrm{ABCD}}$ Means in the same row followed by different uppercase letters are significantly different $(P<0.05)$.

${ }^{1}$ Results are expressed as mean \pm standard error of means; each data point is the average of 3 repeated measurements from 2 independently replicated experiments, $\mathrm{n}=2$.

bation, followed by a rapid decline at the end of the 2$\mathrm{h}$ incubation period. L. casei CSCC 2607, being the most acid-sensitive strain, showed a total reduction of 6.31 log cycles over the 2 -h incubation period, with $4.20 \mathrm{log}$ cycle reduction, mainly in the second hour of incubation.

\section{Bile Tolerance of Lactobacilli}

The effects of oxgall, cholic acid, and taurocholic acid on the growth of lactobacilli are presented in Table 2. Growth of lactobacilli in MRS broth without bile was used as a control. Cholic acid was used as the deconjugated bile, taurocholic acid as the conjugated bile, and oxgall contained both conjugated and deconjugated bile. Due to the addition of bile acids to the media, the effect of $\mathrm{pH}$ was monitored. Generally, media containing cholic acid had lower $\mathrm{pH}$ values compared with the other media. However, the reduction in $\mathrm{pH}$ values due to the production of acid by these lactic acid bacteria was more likely to be strain-dependent. The largest $\mathrm{pH}$ decrease of the media occurred with strains $L$. casei ASCC 290 and CSCC 2607 incubated in media supplemented with oxgall. The average reduction was highest from media supplemented with oxgall (0.81), despite the fact that media with cholic acid had the lowest initial $\mathrm{pH}$ values, whereas the lowest $\mathrm{pH}$ reduction was from media supplemented with taurocholic acid (0.11). These findings indicated that the initial $\mathrm{pH}$ of media with different bile sources had minimal influence toward subsequent growth and bile tolerance of the cultures, and $\mathrm{pH}$-related inhibitory actions of bile salts. Most strains of $L$. acidophilus showed better growth in MRS broth without bile compared with those of $L$. casei. Lactobacillus acidophilus ATCC 4356 showed similar growth in the presence of bile salt (oxgall and cholic acid) and without bile, whereas the growth of $L$. casei ASCC 290 was inhibited in the presence of oxgall compared with the control. All strains showed faster growth in MRS broth containing cholic acid; slower growth was observed in the presence of taurocholic acid. Lactobacillus casei ASCC 1520, L. casei ASCC 1521, L. casei ASCC 290, L. casei ATCC 15820, and L. acidophilus ATCC 4357 were most bile-tolerant in the presence of cholic acid, whereas L. acidophilus ATCC 4356 and L. casei CSCC 2607 were the least tolerant. Most strains of $L$. casei showed better tolerance to cholic acid compared with those of $L$. acidophilus. In the presence of taurocholic acid, strains of $L$. acidophilus showed overall greater tolerance compared with those of $L$. casei. However, when comparing control and taurocholic acid on the average, there was a 1.37 -h difference with $L$. acidophilus (principally due to strain 4356), compared with only $1.06 \mathrm{~h}$ with $L$. casei. We postulate that the slow growth of $L$. casei in the presence of taurocholic acid may have been influenced by its slower growth in MRS than by inhibition effects of conjugated bile. L. acidophilus ATCC 33200, L. acidophilus ATCC 4357, L. acidophilus ATCC 4962, and L. casei ASCC 1521 were most tolerant toward taurocholic acid, and $L$. casei ASCC 279 was the least tolerant strain $(P<0.05)$. L. casei and $L$. acidophilus showed various levels of tolerance to oxgall; L. casei ASCC 1520, L. casei ASCC 292, L. casei ATCC 15820, and L. acidophilus ATCC 33200 showed better tolerance than $L$. casei ASCC 1521, $L$. casei ASCC 279, and L. casei ASCC 290.

\section{Cholesterol Assimilation by Lactobacilli}

Levels of cholesterol assimilation during $20 \mathrm{~h}$ of growth of the strains are shown in Table 3. Cholesterol removal varied among strains $(P<0.05)$ and ranged from 3.76 to $34.69 \mu \mathrm{g} / \mathrm{mL}$. In general, both $L$. acido- 

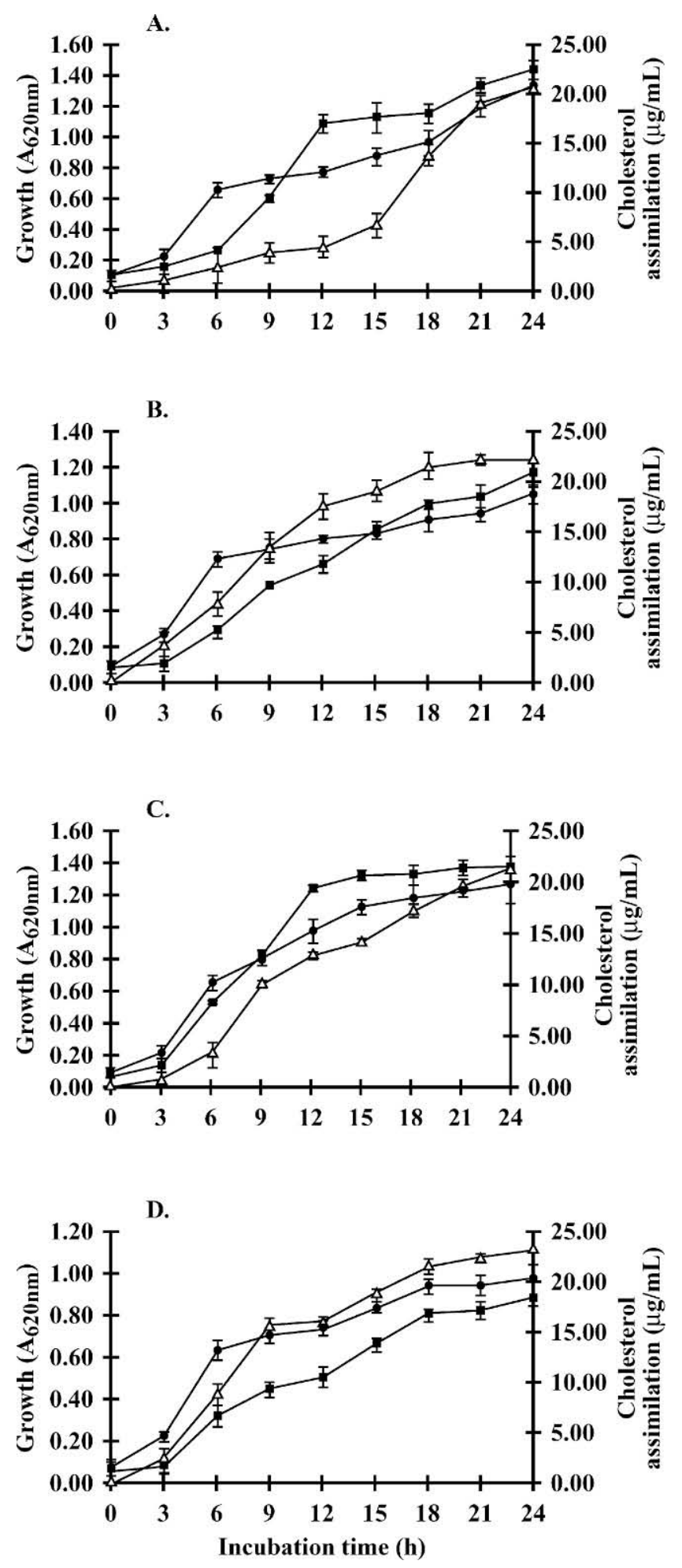

Figure 1. Growth profiles in medium with cholesterol (ם) and without cholesterol $(\bullet)$, and cholesterol assimilation patterns $(\triangle)$ for Lactobacillus acidophilus ATCC 33200 (A), L. acidophilus ATCC 4356 (B), L. acidophilus ATCC 4357 (C), and L. acidophilus ATCC 4962 (D). Error bars represent SEM; each data point is the average of 2 repeated measurements from 2 independently replicated experiments, $\mathrm{n}=2$. philus and L. casei assimilated more cholesterol in the presence of cholic acid compared with the control. In broth containing cholic acid, overall cholesterol removal was observed to be higher for strains of $L$. casei compared with those of L. acidophilus. L. casei ASCC 1520, L. casei ASCC 1521, L. casei ASCC 292, L. casei ATCC 15820, and L. acidophilus ATCC 4962 assimilated more than $25 \mu \mathrm{g} / \mathrm{mL}$ cholesterol in the presence of cholic acid, and were better than $L$. acidophilus ATCC 4356, $L$. acidophilus ATCC 4357, and L. casei CSCC 2607, which assimilated less than $15 \mu \mathrm{g} / \mathrm{mL}$ cholesterol. In broth containing taurocholic acid, the lowest cholesterol assimilation was observed for most strains of lactobacilli studied compared with the control. Strains of $L$. acidophilus ATCC 33200 and L. casei ASCC 1521 removed the highest level of cholesterol (more than $20 \mu \mathrm{g} / \mathrm{mL}$ ), however, the level was lower than other bile salts studied $(P<0.05)$. Cholesterol assimilation by strains of $L$. acidophilus was significantly higher than that of $L$. casei in the presence of taurocholic acid. Cholesterol assimilation in broth containing oxgall varied from 12.03 to $32.25 \mu \mathrm{g} / \mathrm{mL}$. Most strains were able to remove a higher level of cholesterol compared with the control, except for strains of $L$. acidophilus ATCC 4357, L. casei ASCC 279, and L. casei ASCC 290. Cholesterol assimilation may be influenced by biomass levels that were not identical in the various treatments. Thus, growth and cholesterol assimilation patterns were further studied.

\section{Growth and Cholesterol Assimilation Patterns}

Growth of all lactobacilli studied in the presence or absence of cholesterol, and the cholesterol assimilation patterns are illustrated in Figures 1 and 2. Oxgall was chosen instead of cholic acid because it more closely represents the human gut model, although the cholic acid produced higher growth and cholesterol assimilation. Most strains exhibited higher growth in the presence of cholesterol with the exception of strain $L$. acidophilus ATCC 4962 (Figure 1D), ASCC L. casei 1520 (Figure 2A) and L. casei ASCC 1521 (Figure 2B), which showed better growth in the absence of cholesterol. Most strains had higher growth for the first 9 to $15 \mathrm{~h}$ in the medium containing no cholesterol followed by slower growth until the end of the 24-h incubation period. In the presence of cholesterol, most strains showed gradual growth for the first 12 to $18 \mathrm{~h}$, followed by rapid growth thereafter. Cholesterol assimilation patterns varied with strains studied. Cholesterol assimilation patterns and growth curves indicated that cholesterol removal was growth-associated. 
A

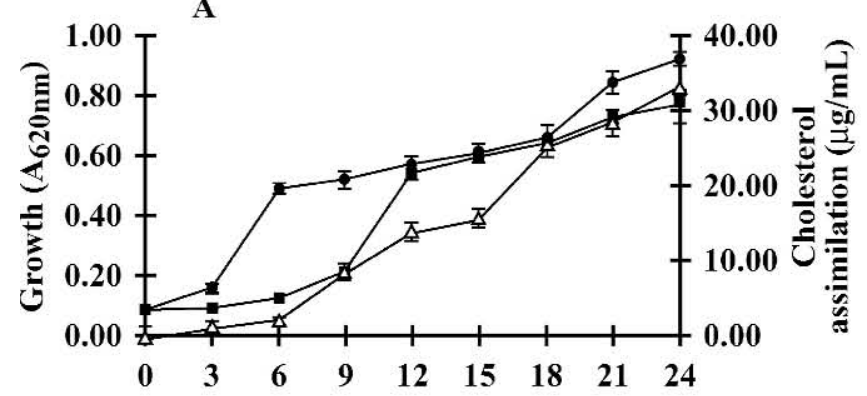

C

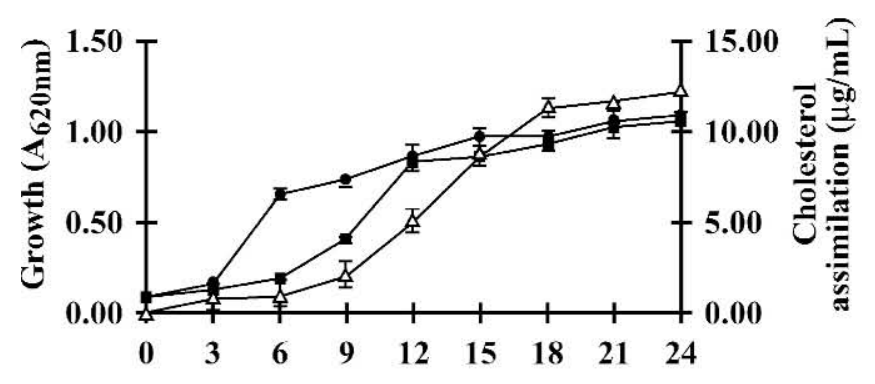

E
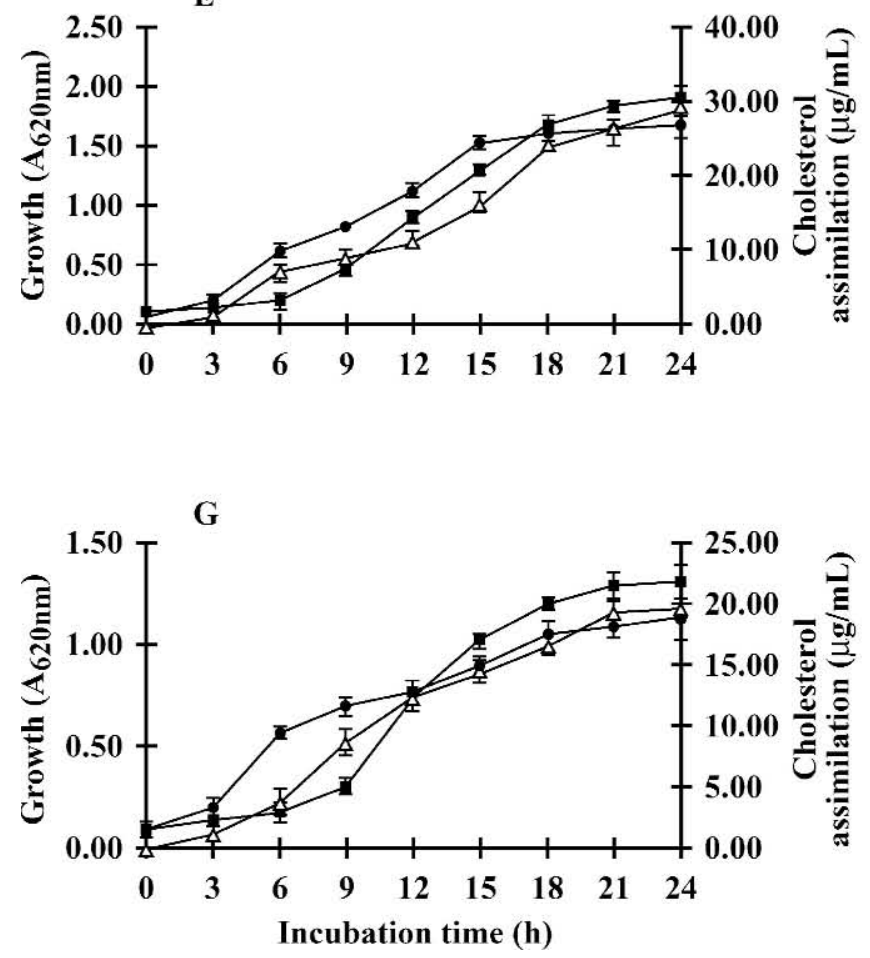

B

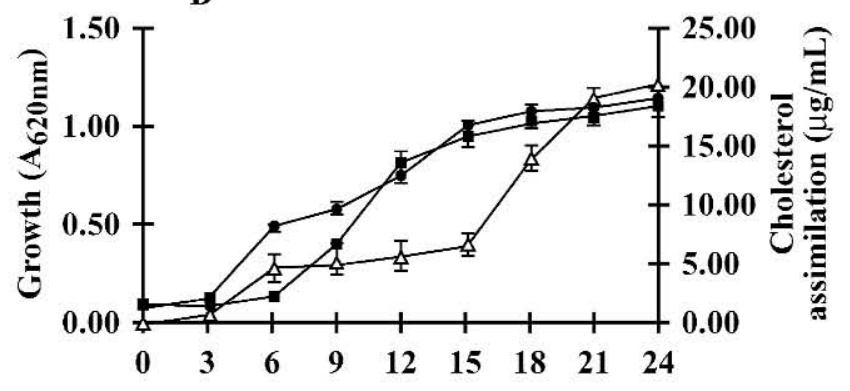

D

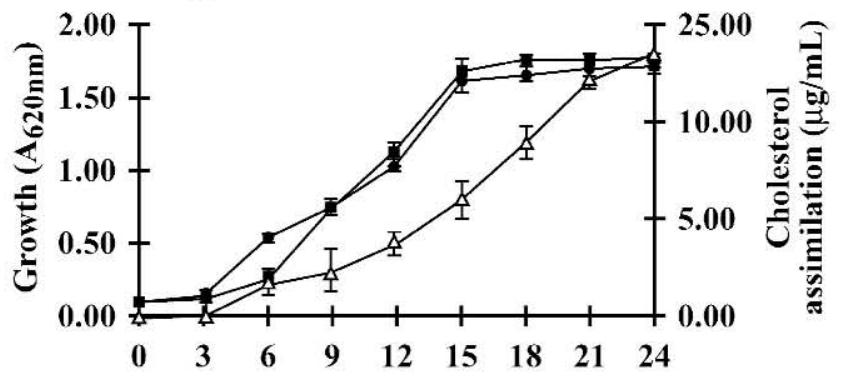

$\mathbf{E}$

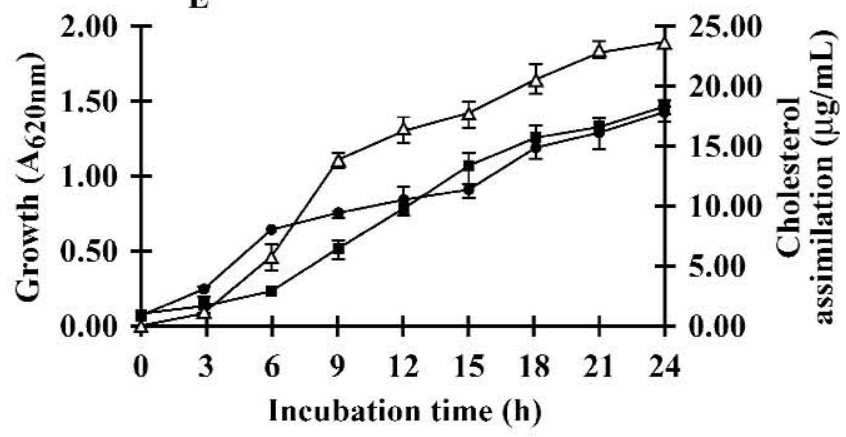

Figure 2. Growth profiles in medium with cholesterol (ם), and without cholesterol $(\bullet)$, and cholesterol assimilation patterns $(\triangle)$ for Lactobacillus casei ASCC 1520 (A), L. casei ASCC 1521 (B), L. casei ASCC 279 (C), L. casei ASCC 290 (D), L. casei ASCC 292 (E), L. casei ATCC $15820(\mathrm{~F})$, and L. casei CSCC 2607 (G). Error bars represent SEM; each data point is the average of 2 repeated measurements from 2 independently replicated experiments, $\mathrm{n}=2$. 


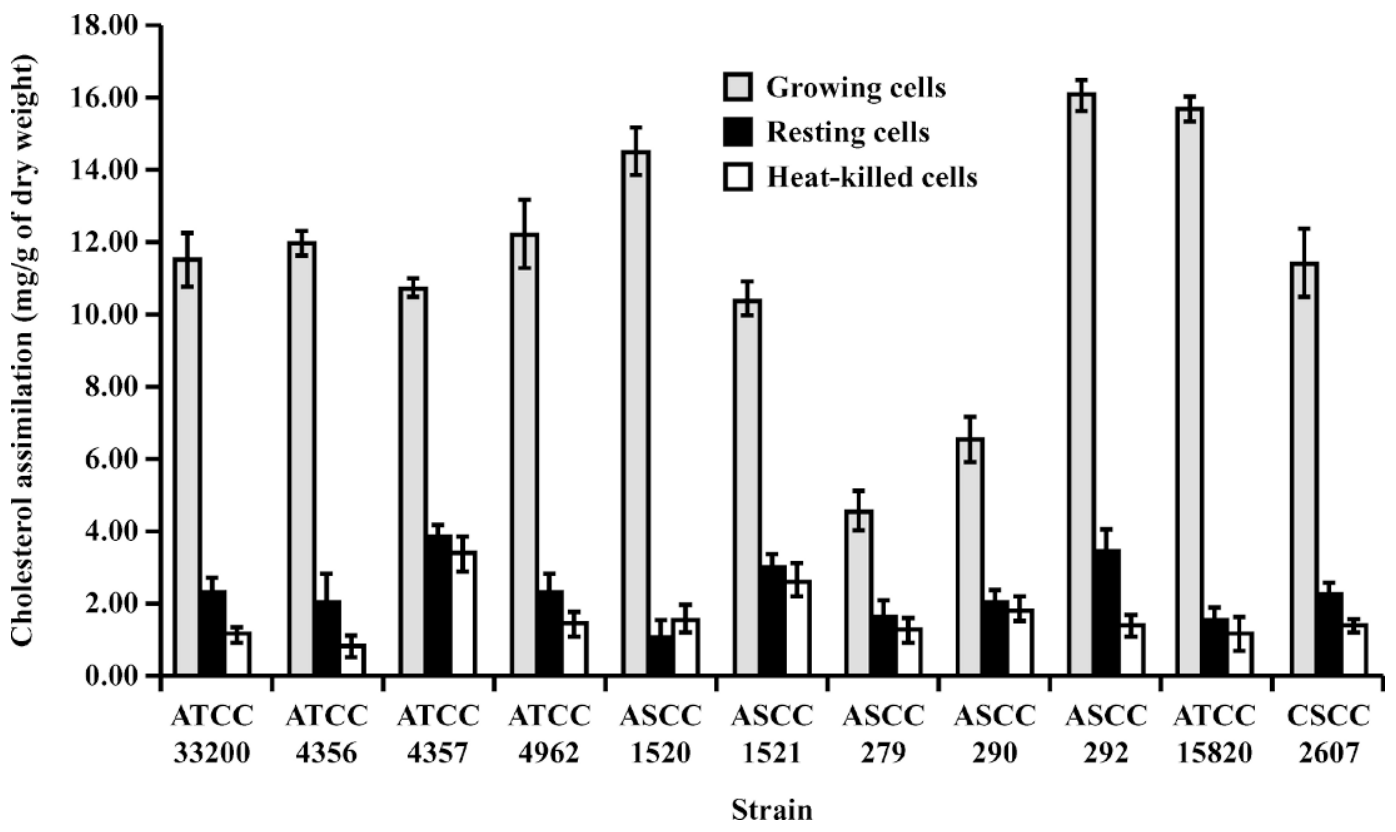

Figure 3. Cholesterol removal by growing, resting, and heat-killed cells of Lactobacillus acidophilus ATCC 33200, L. acidophilus ATCC 4356, L. acidophilus ATCC 4357, L. acidophilus ATCC 4962, L. casei ASCC 1520, L. casei ASCC 1521, L. casei ASCC $279, L$. casei ASCC 290, L. casei ASCC 292, L. casei ATCC 15820, and L. casei CSCC 2607 (from left to right). Cholesterol assimilation $=\left(\mathrm{C}_{1}-\mathrm{C}_{2}\right) /\left(\mathrm{W}_{2}-\mathrm{W}_{1}\right)$, where $\mathrm{C}_{1}$ and $\mathrm{C}_{2}$ were the amount of cholesterol present in the fermentation broths at time $=0$ and time $=20 \mathrm{~h}$ respectively, and $\mathrm{W}_{1}$ and $\mathrm{W}_{2}$ were the dry weight of the individual culture at time $=0$ and time $=20 \mathrm{~h}$ respectively, for all treatments studied. Error bars represent SEM; each data point is the average of 2 repeated measurements from 2 independently replicated experiments, $n=2$.

\section{Cholesterol Removal by Growing, Dead, and Resting Cells}

We wanted to find out whether nongrowing cells could remove cholesterol. The amount of cholesterol removed by growing, resting, and dead cells is illustrated in Figure 3. Heat-killed and resting cells showed a small degree of cholesterol removal, ranging from 0.79 to 3.82 $\mathrm{mg} / \mathrm{g}$ of dry weight, compared with 4.53 to $16.03 \mathrm{mg} / \mathrm{g}$ of dry weight for growing cells. Although there was no significant difference between cholesterol removal by resting and dead cells, most strains exhibited higher cholesterol removal when cells were resting suspended in phosphate buffer ( $\mathrm{pH}$ 6.8), compared with heat-killed cells, except for L. casei ASCC 1520, which removed more cholesterol when cells were killed than when resting. L. casei ASCC 292, L. casei ATCC 15820, and $L$. casei ASCC 1520 assimilated more cholesterol during growth, but not when resting or killed. On the other hand, L. acidophilus ATCC 4357 and L. casei ASCC 1521 assimilated less cholesterol during growth but showed relatively higher cholesterol assimilation when cells were resting and dead. $L$. casei ASCC 279 and $L$. casei ASCC 290 assimilated the lowest level of cholesterol during growth.

\section{Effect of Cholesterol on Cellular Fatty Acid Composition}

Effect of cholesterol on cellular fatty acid compositions is presented in Tables 4 and 5. Cellular fatty acid patterns varied between strains grown with or without cholesterol. Strains grown in the absence of cholesterol had higher percentages of oleic and linoleic acids compared with those grown in the presence of cholesterol. Cellular fractions of cells grown in the presence of cholesterol showed relatively higher percentage of hexadecanoic and octadecanoic acids compared with cells grown in medium without cholesterol. All strains grown in the medium with or without cholesterol showed a higher percentage of total saturated fatty acids than unsaturated fatty acids. Total saturated fatty acids were higher in cellular fractions of cells grown in the presence of cholesterol, while total unsaturated fatty acids were lower. However, ATCC L. acidophilus 4356 and L. acidophilus 4357 showed lower total level of saturated fatty acids per gram of dry weight of cells when grown in the medium with cholesterol compared with that without cholesterol. Strains $L$. casei ATCC 4962, L. casei 15820, L. casei ASCC 1520, L. casei ASCC 1521, L. casei ASCC 290, L. casei ASCC 292, and L. casei CSCC 2607 showed higher cellular total unsatu- 
Table 4. Fatty acid composition of Lactobacillus acidophilus strains grown in medium with or without cholesterol. ${ }^{1}$

\begin{tabular}{|c|c|c|c|c|c|c|c|c|c|}
\hline \multirow[b]{2}{*}{ Fatty acid ${ }^{2}$} & \multirow[b]{2}{*}{ Medium } & \multicolumn{2}{|c|}{ ATCC 33200} & \multicolumn{2}{|c|}{ ATCC 4356} & \multicolumn{2}{|c|}{ ATCC 4357} & \multicolumn{2}{|c|}{ ATCC 4962} \\
\hline & & $\%^{3}$ & $\mathrm{mg} / \mathrm{g}^{4}$ & $\%$ & $\mathrm{mg} / \mathrm{g}$ & $\%$ & $\mathrm{mg} / \mathrm{g}$ & $\%$ & $\mathrm{mg} / \mathrm{g}$ \\
\hline \multirow[t]{2}{*}{ 10:0 } & Cholesterol & 9.39 & 1.11 & 9.88 & 1.14 & 7.99 & 0.75 & 10.94 & 1.35 \\
\hline & Without cholesterol & 7.16 & 0.59 & 7.11 & 1.02 & 6.78 & 1.56 & 7.99 & 0.39 \\
\hline \multirow[t]{2}{*}{$12: 0$} & Cholesterol & 10.66 & 1.11 & 11.42 & 1.16 & 9.83 & 0.81 & 11.18 & 1.21 \\
\hline & Without cholesterol & 7.58 & 0.55 & 8.47 & 1.07 & 8.32 & 1.69 & 9.52 & 0.41 \\
\hline \multirow[t]{2}{*}{$14: 0$} & Cholesterol & 11.61 & 1.10 & 12.53 & 1.16 & 10.45 & 0.79 & 12.19 & 1.21 \\
\hline & Without cholesterol & 8.24 & 0.54 & 9.41 & 1.09 & 9.41 & 1.74 & 10.05 & 0.39 \\
\hline \multirow[t]{2}{*}{$16: 0$} & Cholesterol & 20.12 & 1.80 & 18.39 & 1.60 & 20.13 & 1.43 & 18.66 & 1.74 \\
\hline & Without cholesterol & 15.71 & 0.98 & 18.33 & 1.99 & 18.18 & 3.17 & 17.38 & 0.64 \\
\hline \multirow[t]{2}{*}{$16: 1$} & Cholesterol & 7.20 & 0.67 & 8.06 & 0.72 & 8.61 & 0.63 & 7.80 & 0.75 \\
\hline & Without cholesterol & 6.01 & 0.39 & 5.96 & 0.67 & 6.30 & 1.14 & 6.94 & 0.26 \\
\hline \multirow[t]{2}{*}{ 18:0 } & Cholesterol & 14.59 & 1.25 & 13.97 & 1.16 & 15.07 & 1.02 & 14.08 & 1.25 \\
\hline & Without cholesterol & 11.86 & 0.70 & 12.98 & 1.34 & 12.37 & 2.06 & 14.30 & 0.50 \\
\hline \multirow[t]{2}{*}{$18: 1$} & Cholesterol & 10.12 & 0.87 & 8.70 & 0.73 & 9.55 & 0.65 & 8.61 & 0.77 \\
\hline & Without cholesterol & 18.98 & 1.13 & 16.66 & 1.74 & 18.67 & 3.13 & 14.36 & 0.51 \\
\hline \multirow[t]{2}{*}{$18: 2$} & Cholesterol & 8.49 & 0.69 & 8.74 & 0.69 & 9.51 & 0.62 & 8.43 & 0.72 \\
\hline & Without cholesterol & 17.93 & 1.02 & 14.74 & 1.46 & 13.48 & 2.14 & 11.98 & 0.50 \\
\hline \multirow[t]{2}{*}{ Others } & Cholesterol & 7.81 & 1.12 & 8.31 & 1.16 & 8.86 & 1.01 & 8.11 & 1.22 \\
\hline & Without cholesterol & 6.53 & 0.65 & 6.35 & 1.11 & 6.49 & 1.82 & 7.49 & 0.44 \\
\hline \multirow[t]{2}{*}{ SFA } & Cholesterol & 66.38 & 6.37 & 66.19 & 6.21 & 63.47 & 4.79 & 67.05 & 6.76 \\
\hline & Without cholesterol & 50.55 & 3.36 & 56.30 & 6.51 & 55.06 & 10.22 & 59.24 & 2.32 \\
\hline \multirow[t]{2}{*}{ UFA } & Cholesterol & 25.81 & 2.23 & 25.50 & 2.14 & 27.67 & 1.90 & 24.84 & 2.24 \\
\hline & Without cholesterol & 42.92 & 2.54 & 37.35 & 3.86 & 38.46 & 6.41 & 33.27 & 1.17 \\
\hline \multirow{2}{*}{ Total FA } & Cholesterol & & 9.72 & & 9.51 & & 7.70 & & 10.22 \\
\hline & Without cholesterol & & 6.55 & & 11.48 & & 18.45 & & 3.93 \\
\hline
\end{tabular}

${ }^{1}$ Results are expressed as mean \pm standard error of means; each data point is the average of 2 repeated measurements from 2 independently replicated experiments, $\mathrm{n}=2$.

${ }^{2} \mathrm{SFA}=$ Total saturated fatty acids, UFA $=$ total unsaturated fatty acids, Total FA = total saturated, unsaturated, and other fatty acids.

${ }^{3}$ Percentage of total fatty acid content.

${ }^{4}$ Milligrams of fatty acids per gram (dry weight) of cells.

rated fatty acids when cells were grown in the medium with cholesterol compared with those without cholesterol. Most strains showed higher level of total fatty acids when cells were grown in the presence of cholesterol compared with those in its absence.

\section{DISCUSSION}

High level of serum cholesterol has been associated with risks of coronary heart disease. The use of probiotic bacteria in reducing serum cholesterol levels has attracted much attention. Probiotic bacteria are mostly delivered in a food system and must be acid and bile tolerant to survive in the human gastrointestinal tract. The time from entrance to release from the stomach has been estimated to be approximately $90 \mathrm{~min}$, with further digestive processes requiring longer residence time (Berada et al., 1991). Stresses to organisms begin in the stomach, with $\mathrm{pH}$ between 1.5 and 3.0, and in the upper intestine that contains bile (Lankaputhra and Shah, 1995; Corzo and Gilliland, 1999). Survival at $\mathrm{pH} 3.0$ for $2 \mathrm{~h}$ and at a bile concentration of 1000 $\mathrm{mg} / \mathrm{L}$ is considered optimal acid and bile tolerance for probiotic strains (Usman, 1999). Strains of lactobacilli used in this study showed varying levels of viability at $\mathrm{pH} 2.0$ after a 2-h incubation. L. acidophilus ATCC 4357, L. acidophilus ATCC 4962, L. casei ASCC 290, and $L$. casei ASCC 292 survived best under acidic conditions, whereas viability of strains ASCC 1520, ASCC 1521, ASCC 279, ATCC 15820, and CSCC 2607 was greatly reduced. In general, L. acidophilus strains survived better under acidic conditions than $L$. casei.

Growth observed in the presence of different bile sources suggested that conjugated bile (taurocholic acid) was more inhibitory toward strains of lactobacilli compared with deconjugated bile (cholic acid) and oxgall. The resistance of the lactobacilli toward deconjugated bile may be because conjugated bile salts have greater solubility and detergent activity, and may, therefore be more toxic than their deconjugated counterpart. This was supported by the fact that the cholic acid added to the fermentation broths was far less soluble than taurocholic acid, based on the solubility index. Taurocholic acid was not accumulated by Lactobacillus salivarus JCM 1044 due to its hydrophilicity (Kurdi et al., 2000). Such preference was supported by previous reports (Kurdi et al., 2000; Yokota et al., 2000), which showed that Lactobacillus species actively accumulated 
Table 5. Fatty acid composition of Lactobacillus casei strains grown in medium with or without cholesterol. ${ }^{1}$

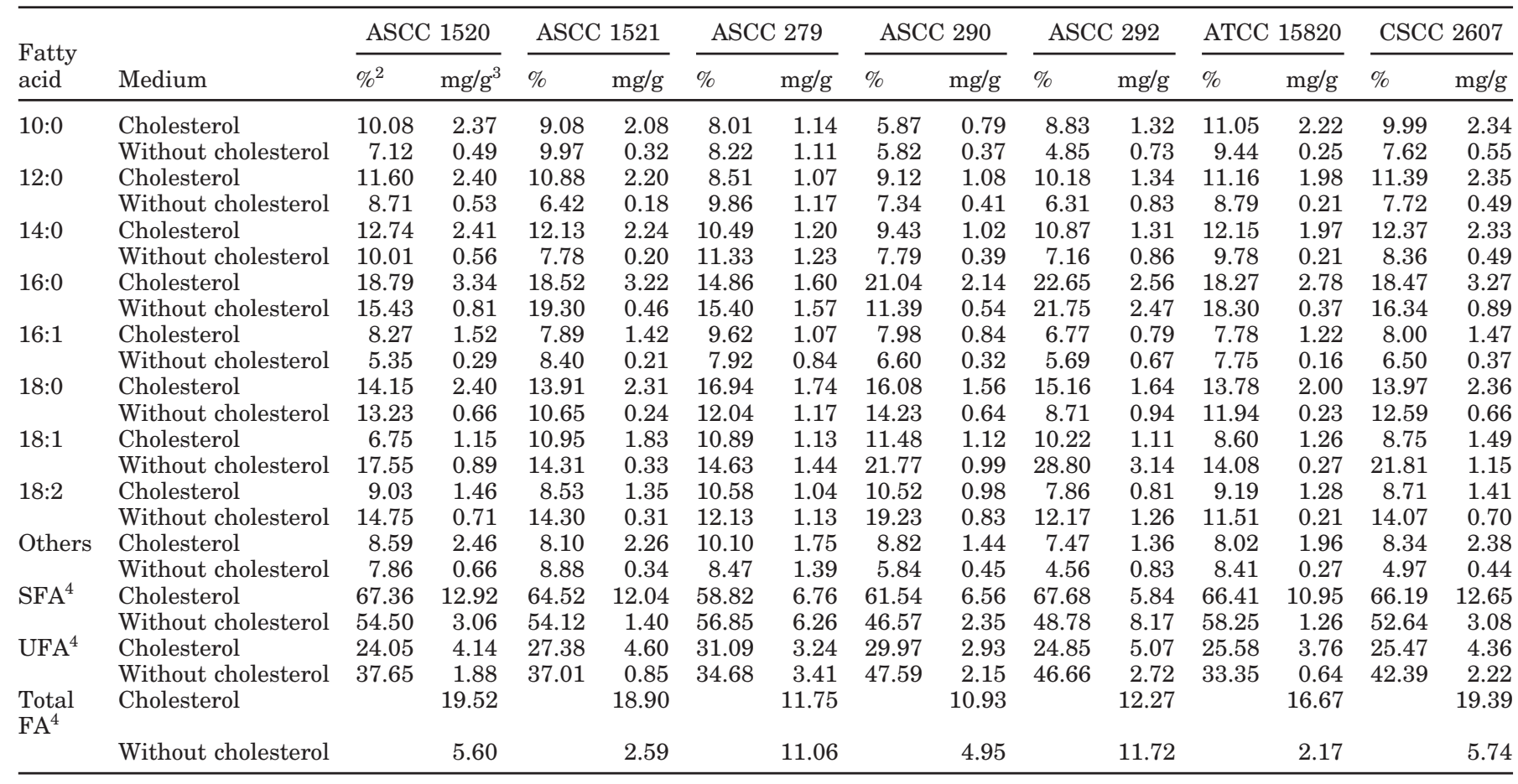

${ }^{1}$ Results are expressed as mean \pm SEM; each data point is the average of 2 repeated measurements from 2 independently replicated experiments, $\mathrm{n}=2$.

${ }^{2}$ Percentage of total fatty acid content.

${ }^{3}$ Milligrams of fatty acids per gram (dry weight) of cells.

${ }^{4} \mathrm{SFA}=$ Total saturated fatty acids, UFA = total unsaturated fatty acids, Total FA = total saturated, unsaturated, and other fatty acids.

cholic acid in an ATP-dependent manner, or when they were energized by glucose. However, results from our study contradicted previous studies that found deconjugated bile salts to have greater tendency to damage the

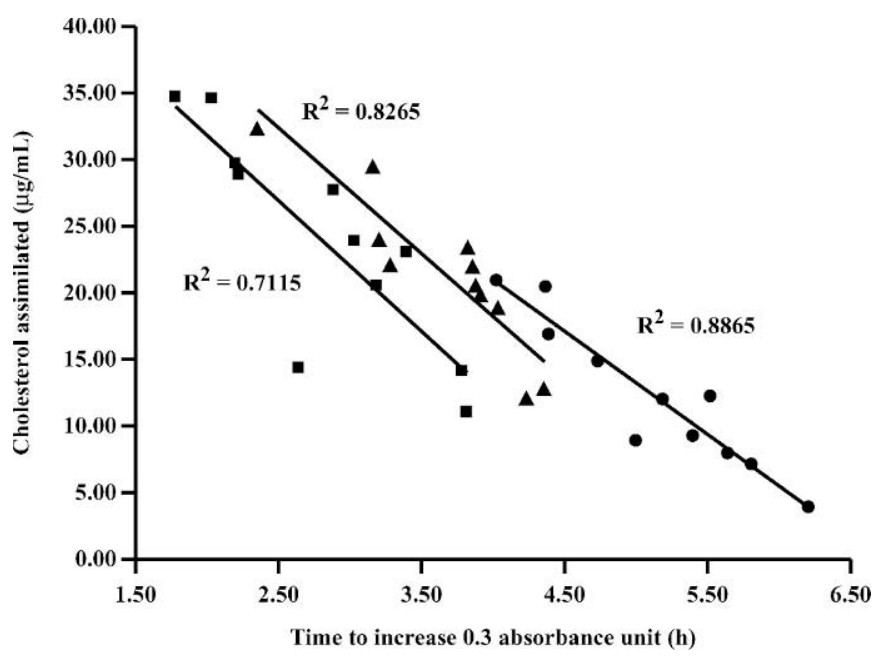

Figure 4. Regression analyses of cholesterol assimilation and bile tolerance in de Man, Rogosa, Sharpe media containing $0.30 \%$ oxgall $(\mathbf{\Delta}), 0.3 \%$ cholic acid (ם), and $0.35 \%$ taurocholic acid $(\bullet)$. cell membrane due to their hydrophobic nature. Free and deconjugated bile acids have the ability to disaggregate the ordered structure of biological membrane and cytoplasmic membrane, respectively. Deconjugated sodium taurocholate was reported to have such properties and therefore, have higher inhibitory effects against bacterial cells (Tannock et al., 1997). Similarly, according to Yokota et al. (2000), the degree of sensitivity of Lactococcus lactis was consistent with the degree of hydrophobicity of the compounds, the highest being for deconjugated bile followed by conjugated one. Our results suggest that under high concentration of conjugated bile, strains of $L$. acidophilus are likely to survive best, whereas in presence of deconjugated bile, L. casei would survive best. Growth in the presence of oxgall showed that L. casei ASCC 1520, L. casei ASCC 290, and $L$. casei ATCC 15820 survived best, whereas $L$. casei ASCC 1521, L. casei ASCC 279, and L. casei ASCC 290 would be least bile tolerant.

Cholesterol assimilation in the presence of different bile sources showed a good relationship $\left(\mathrm{R}^{2}=0.71\right.$ to 0.89 ) with bile tolerance of the strains studied (Figure 4). Strains showing greater tolerance toward deconjugated bile exhibited higher overall cholesterol assimilation in the presence of cholic acid, whereas those show- 
ing greater inhibition by taurocholic acid produced lower cholesterol assimilation. Also, most strains exhibited better growth in the presence of cholesterol, indicating that cholesterol stimulated their growth. Regression analyses suggested that cholesterol removal was closely associated with the bacterial growth. Cholesterol assimilation by growing cells was significantly higher than resting and dead counterparts, however, there was no significant difference $(P<0.05)$ in the level of cholesterol removal by resting and dead cells. The capability of strains in dead and resting stages to remove cholesterol indicated that cholesterol might be removed via binding to cells. Higher cholesterol removal by growing cells indicated that the degree of bound cholesterol might be dependent on the growth of cells. The physiological $\mathrm{pH}$ in the intestinal tract of humans is usually neutral to alkaline (Kimoto et al., 2002). Although cholesterol assimilation occurred mainly with growing cells, results on cholesterol removal from media at $\mathrm{pH} 6.8$ by heat-killed cells indicated the potential of nonviable cells to reduce cholesterol concentration in the gastrointestinal system.

To further examine the possibility of cholesterol incorporation into the membrane fraction of cells, changes in cellular lipid profiles were quantified using a gas chromatograph. Methanolic HCl does not methylate free fatty acids, thus methylated fatty acids were components of the membrane phospholipids. The lipids in gram-positive bacteria are found predominantly in the membrane (Kimoto et al., 2002), and fatty acids in bacteria are primarily the precursors of cellular phospholipids (Li and Cronan, 1993). Thus, cholesterol removed in vitro could be incorporated into the cell membrane, and this may alter the fatty acid profiles, especially hexadecanoic, octadecanoic, total saturated, and unsaturated acids. Such alteration of the cellular envelope was supported by Noh et al. (1997), who found that cells of $L$. acidophilus ATCC 43121 grown in the presence of cholesterol micelles showed greater resistance to lysis by sonication than those grown without cholesterol. Kimoto et al. (2002) found that there was a difference in the fatty acid distribution pattern for Lactococcus lactis cells grown with or without cholesterol, suggesting that the fatty acids composition of the cells was altered because of cholesterol being incorporated into the cellular membrane after its removal from the medium. Our results showed that total fatty acids obtained from most cells grown in the presence of cholesterol were higher than those grown in the absence, with the exception of strains L. acidophilus ATCC 4356 and L. acidophilus ATCC 4357. We postulate that the increment in total fatty acids was contributed by cholesterol incorporation into the membrane and not by cellular synthesis, because lactic acid bacteria growing under high levels of lipid were previously reported to lose the ability to synthesize fatty acids (Kiatpapan et al., 2001).

\section{CONCLUSIONS}

All strains of lactobacilli studied survived the acidic conditions and bile concentration to a variable extent. Similarly, all strains have varying capabilities to remove cholesterol in vitro. Three possible mechanisms for removal of cholesterol from media by lactobacilli are proposed: assimilation of cholesterol during growth, incorporation of cholesterol into the membrane of cells, and binding of cholesterol to the cell surface. L. casei ASCC 292 and L. acidophilus 4962 strains showed good acid and bile tolerance, and highest cholesterol removal from media, indicating that these strains may be promising candidates for use as a dietary adjunct to lower serum cholesterol in vivo.

\section{REFERENCES}

Anderson, J. W., and S. E. Gilliland. 1999. Effect of fermented milk (yogurt) containing Lactobacillus acidophilus L1 on serum cholesterol in hypercholesterolemic humans. J. Am. Coll. Nutr. 18:43-50.

Berada, N., J. F. Lemeland, G. Laroch, P. Thouvenot, and M. Piala. 1991. Bifidobacterium from fermented milks: Survival during gastric transit. J. Dairy Sci. 74:409-413.

Bermudez, L., C. B. Inderlied, P. Kolonoski, M. Wu, P. Aralar, and L. S. Young. 2001. Telithromycin is active against Mycobacterium avium in mice despite lacking significant activity in standard in vitro and macrophage assays and is associated with low frequency of resistant during treatment. Antimicrob. Agents Chemother. 45:2210-2214.

Brashears, M. M., S. E. Gilliland, and L. M. Buck. 1998. Bile salt deconjugation and cholesterol removal from media by Lactobacillus casei. J. Dairy Sci. 81:2103-2110.

Corzo, G., and S. E. Gilliland. 1999. Measurement of bile salt hydrolase activity from Lactobacillus acidophilus based on disappearance of conjugated of bile salts. J. Dairy Sci. 82:466-471.

Dambekodi, P. C., and S. E. Gilliland. 1998. Incorporation of cholesterol into the cellular membrane of Bifidobacterium longum. J. Dairy Sci. 81:1818-1824.

Fuller, R. 1992. Probiotics: The scientific basis. Chapman and Hall, London, UK.

Gibson, G. R., P. B. Otaway, and A. R. Robert. 2000. Prebiotics: New Developments in Functional Foods. Chandos Publishing (Oxford) Limited, London, UK.

Gilliland, S. E., and D. K. Walker. 1990. Factors to consider when selecting a culture of $L$. acidophilus as a dietary adjunct to produce a hypercholesterolemic effect in humans. J. Dairy Sci. 73:905-909.

Greenwald, C. G. 1991. Overview of fat and cholesterol reduction technologies. Pages 21-34 in Fat and Cholesterol Reduced Foods. C. Haberstroh and C. E. Morris, ed. Gulf Publishing, London, UK.

Kiatpapan, P., H. Kobayashi, M. Sakaguchi, H. Ono, M. Yamashita, Y. Kaneko, and Y. Morooka. 2001. Molecular characterization of Lactobacillus plantarum genes for $\beta$-ketoacyl-acyl carrier protein synthase III $(f a b H)$ and acetyl coenzyme A carboxylase ( $a c c B$ $C D A$ ), which are essential for fatty acid biosynthesis. App. Environ. Microbiol. 67:426-433.

Kimoto, H., S. Ohmomo, and T. Okamoto. 2002. Cholesterol removal from media by lactococci. J. Dairy Sci. 85:3182-3188.

Kurdi, P., H. W. Van Veen, H. Tanaka, I. Mierau, W. N. Konings, G. W. Tannock, F. Tomita, and A. Yokota. 2000. Cholic acid is 
accumulated spontaneously, driven by membrane $\mathrm{pH}$ in many lactobacilli. J. Bacteriol. 182:6525-6528.

Lankaputhra, W. E. V., and N. P. Shah. 1995. Survival of Lactobacillus acidophilus and Bifidobacterium spp. in the presence of acid and bile salts. Cult. Dairy Prod. J. 30:2-7.

Li, S. J., and J. E. Cronan, Jr. 1993. Growth rate regulation of Escherichia coli acetyl coenzyme A carboxylase, which catalyzes the first step of lipid biosynthesis. J. Bacteriol. 175:332-340.

Mann, G. V. 1974. Studies of a surfactant and cholesterolemia in the Masai. Am. J. Clin. Nutr. 27:464-469.

Manson, J. E., H. Tosteson, P. M. Ridker, S. Satterfield, P. Herbert, and G. T. O'Conner. 1992. The primary prevention of myocardial infarction. N. Engl. J. Med. 326:1406-1416.

Murga, M. L. F., G. M. Cabrera, G. F. Valdez, S. Disalvo, and A. M. Seldes. 2000. Influence of growth temperature on cryotolerance and lipid composition of Lactobacillus acidophilus. J. Appl. Microbiol. 88:342-348.

Noh, D. O., S. H. Kim, and S. E. Gilliland. 1997. Incorporation of cholesterol into the cellular membrane of Lactobacillus acidophilus ATCC 43121. J. Dairy Sci. 80:3107-3113.

Pereira, D. I. A., and G. R. Gibson. 2002. Cholesterol assimilation by lactic acid bacteria and bifidobacteria isolated from the human gut. Appl. Environ. Microbiol. 68:4689-4693.

Piston, R. L., and S. E. Gilliland. 1994. Influence of frozen and subsequent refrigerated storage in milk on ability of $L$. acidophilus to assimilate cholesterol. Cult. Dairy Prod. J. 29:9-19.
Rasic, J. L., I. F. Vujicic, M. Skrinjar, and M. Vulic. 1992. Assimilation of cholesterol by some cultures of lactic acid bacteria and bifidobacteria. Biotechnol. Lett. 14:39-44.

Reynier, M. O., J. C. Montet, A. Gerolami, C. Marteau, C. Crotte, A. M. Montet, and S. Mathieu. 1981. Comparative effects of cholic, chenodeoxycholic and ursodeoxycholic acids on micellar solubilization and intestinal absorption of cholesterol. J. Lipid Res. 22:467-473.

Rudel, L. L., and M. D. Morris. 1973. Determination of cholesterol using o-phthalaldehyde. J. Lipid Res. 14:364-366.

Sanders, M. E. 2000. Considerations for use of probiotic bacteria to modulate human health. J. Nutr. 130:384S-390S.

Shaper, A. G., K. W. Jones, and J. Kyobe. 1963. Serum lipids in the three nomadic tribes of Northern Kenya. Am. J. Clin. Nutr. 13:135-146.

Tahri, K., J. P. Grill, and F. Schneider. 1996. Bifidobacteria strain behavior towards cholesterol: Coprecipitation with bile salts and assimilations. Curr. Microbiol. 33:187-193.

Tannock, G. W., G. M. Bateup, and H. F. Jenkinson. 1997. Effect of sodium taurocholate on the in-vitro growth of lactobacilli. Microbiol. Ecology. 33:163-167.

Usman, H. A. 1999. Bile tolerance, taurocholate deconjugation, and binding of cholesterol by Lactobacillus gasseri strains. J. Dairy Sci. 82:243-248.

Yokota, A., M. Veenstra, P. Kurdi, H. W. van Veen, and W. N. Konings. 2000. Cholate resistance in Lactococcus lactis is mediated by an ATP-dependent multispecific organic anion transporter. J. Bacteriol. 182:5196-5201. 